\title{
Du geste musical au traitement numérique du signal
}

\author{
Didier Demigny \\ IUT de Lannion \\ Département réseaux \& télécommunications \\ Email : didier.demigny@univ-rennes1.fr \\ Av. Edouard Branly BP 30219 \\ 22302 Lannion Cedex
}

\author{
Michel Lemeu \\ Collège Paul Le Flem \\ Email : michel.lemeu@ac-rennes.fr \\ 10 , rue du collège \\ 22560 Pleumeur Bodou
}

\begin{abstract}
Résumé-Cet article décrit un ensemble de cinq TP de traitement numérique du signal ayant comme fil conducteur des applications en analyse et production de gestes musicaux. Ils ont été élaborés avec deux objectifs. Le premier et le principal est de tenter de remotiver des étudiants qui pour certains (en nombre non négligeable) ont décidé de baisser les bras devant les matières réputées consommatrices de mathématiques. Le second est de préparer les étudiants qui souhaitent s'orienter vers des masters ou des écoles d'ingénieurs en donnant du sens physique aux expressions mathématiques manipulées. L'introduction décrit le public étudiant du département d'IUT "Réseaux \& Télécommunications", et la nécessité de développer une nouvelle pédagogie. La seconde section montre la genèse de ce couplage musique / traitement numérique du signal (TS). La troisième section décrit les cinq TP en précisant leurs objectifs musicaux et signal. La quatrième section analyse les résultats de l'enquête menée auprès des étudiants sur le ressenti de l'ensemble cours, TD, TP de traitement numérique du signal et propose quelques pistes complémentaires qui seront mises en ouvre l'an prochain. On trouvera en annexe le texte et le corrigé du TP portant sur la translation de spectres. L'ensemble des textes de TP est disponible sur mon blog professionnel[8] avec aussi des exemples de sons traités.
\end{abstract}

\section{INTRODUCTION}

\section{A. Des étudiants qui démathent}

A l'IUT de Lannion, les étudiants du département Réseaux \& Télécommunications ont du talent. Très vite ils développent une forte motivation, des capacités et une réelle autonomie dans la maîtrise de la gestion des systèmes complexes que sont les réseaux et les systèmes de télécommunications.

Par contre, ils éprouvent des difficultés dans toutes les matières utilisatrices de mathématiques. Ces difficultés réelles les empêchent de concevoir les mathématiques comme un outil et freinent leur motivation et leur implication : quoi faire d'un levier qu'on ne peut pas soulever?

\section{B. Un programme pédagogique national ambitieux}

La lecture du programme de mathématique explique une partie des difficultés. Il semble construit pour amener toutes les justifications théoriques nécessaires aux disciplines plus applicatives (transformée de laplace pour l'étude de filtres en transitoire, transformée en $\mathrm{Z}$ pour le traitement numérique du signal,etc). Or il est clair qu'on peut facilement dans une première approche se passer de ces justifications théoriques qui ne sont utiles que lorsqu'on est apte à s'en servir comme levier, mais qui perturbent l'étudiant un peu faible. Plus grave, l'utilisation de cet outil mathématique non indispensable dans le cours applicatif effraie l'étudiant qui ainsi se décourage et se démotive pour une matière dans laquelle tout ne se réduit pas aux mathématiques (électronique, modulations, traitement du signal ...). En balayant le programme de mathématique du DUT R\&T, je me suis rendu compte que dans mon passé d'enseignant-chercheur, je n'ai pas utilisé la moitié des outils proposés. Nous nous trouvons en fait dans la situation du bricoleur qui passe son temps à acheter des outils de bricolage très perfectionnés et très coûteux pour les ranger dans son établi et rarement les utiliser. Peut-être même que le temps passé et les échecs successifs à essayer d'utiliser l'un d'entre eux le dégoûtera du bricolage!

\section{De la nécessité d'apprendre autrement}

Il ne s'agit pas de renoncer à l'usage des mathématiques, mais de construire en n'utilisant que les outils les plus indispensables, les plus simples et les plus appropriés. Ces outils doivent être utilisés le plus souvent possible durant la formation. Il me paraît essentiel aussi dans les matières applicatives de ne pas négliger l'aspect fonctionnel mais au contraire de s'appuyer dessus pour justifier les besoins, les objectifs de dimensionnement. Il faut aussi extraire le maximum d'exemples d'applications de la vie courante ou de champs applicatifs favorisant l'enrichissement culturel. En ce qui concerne le traitement du signal, j'ai choisi de concevoir en premier les travaux pratiques, puis des travaux dirigés conduisant à partir d'exercices simples à la préparation des TP puis de rédiger des cours qui ne présentent que les fondamentaux largement illustrés d'exemples.

Le présent papier présente la construction d'une série de travaux pratiques dont le fil rouge ludique est la production d'effets musicaux qui permettent de balayer les fondamentaux du traitement numérique du signal. 


\section{LE PROJET MUSIQUE \& TNS}

\section{A. Le geste musical}

Lors de l'événement européen "la nuit des chercheurs" en 2007 à Lannion, Michel Lemeu ${ }^{1}$ [1] organise un concert commenté de musique synthétique en proposant notamment l'analyse d'un morceau de SUD œuvre de Jean Claude Risset[2] (médaille d'or du CNRS en 1999). M. Lemeu décrit alors la notion de geste musical : un processus créatif qui remplit l'espace entre l'intention musicale et les sons musicaux, processus qui inclut le passage de l'intention musicale à l'onde physique mais aussi de l'onde physique à la perception de l'auditeur. M. Lemeu présente à titre d'exemple :

- la superposition d'un son original et de ce même son décalé temporellement dont l'archétype n'est autre que le canon (Frère Jacques ...) mais qui permet aussi grâce à des décalages temporels plus faibles de colorer des sons naturels qui semblent alors portés par une note de récitation;

- la superposition d'un son original et de ce même son décalé en fréquence dont l'archétype est l'organum datant du haut moyen-âge qui donne naissance (pour peu qu'on réduise à quelques dixièmes de Hertz l'écart en fréquence) aux effets de phasing ou de flange des synthétiseurs modernes.

M. Lemeu montre alors (traitement du son en direct et écoute) comment Jean Claude Risset a utilisé ces deux gestes musicaux dans son œuvre SUD pour transformer une matière sonore naturelle "mer-mistral-galets" [3] (noté MMG dans la suite de ce papier).

Cette expérience a été le facteur déclenchant du remaniement des TP de traitement numérique de signal basé sur quatre constats : (a) le résultat sonore est saisissant et devrait étonner les étudiants, (b) c'est une application ludique du TNS qui n'a rien à voir avec les télécoms et les réseaux qui enrichirait les connaissances générales des étudiants, (c) les traitements à effectuer sans être rudimentaires semblent relativement peu complexes, (d) tous les PC du département sont équipés de "carte sons", du logiciel Matlab et nous disposons de casques en nombre suffisant...

Avant de réaliser la conception de ces TP, il reste deux questions importantes à traiter. D'abord, ce thème est-il suffisamment riche pour illustrer la totalité du programme? Puis, le signal naturel utilisé "MMG" est-il bien adapté à l'enseignement? i.e. les étudiants (et les enseignants) pourront-ils aisément interpréter les observations temporelles et spectrales et effectuer sur ces observations des mesures simples?

\section{B. Mise en musique des cinq TP de traitement numérique du signal}

1) de la mer à la guitare: La réponse à la seconde question est non. Le signal naturel "MMG" a un spectre continu et

\footnotetext{
${ }^{1}$ Michel LEMEU est professeur agrégé d'Education Musicale et chant choral au Collège Paul Le Flem de Pleumeur Bodou; Chargé d'enseignement d'informatique musicale à l'Université Rennes 2; Interlocuteur Académique TICE et Education Musicale pour l'Académie de Rennes.
}

très variable dans le temps. Il ne permet pas de comprendre facilement par l'observation spectrale ou temporelle les transformations opérées par les "gestes musicaux". J'ai donc choisi d'utiliser comme sons bruts des notes "pures" de guitare (quasiment un spectre de raies) que j'ai collectées sur le net [4] : LA $3^{\grave{e ̀ m e}}$ octave $(440 \mathrm{~Hz}), \mathrm{RE \#} 4^{\text {ème }}$ octave $(620 \mathrm{~Hz})$, etc.

Ces notes sont disponibles sous forme de fichiers au format "wav" échantillonnées à $44,1 \mathrm{KHz}$ et chacune d'une durée de l'ordre de 2 secondes. On montrera dans la section III au sujet du TP1 que l'analyse de ces sons plus simples peut malgré tout réserver quelques surprises. Le son MMG est aussi mis à disposition des étudiants afin de reproduire les gestes musicaux mis en œuvre par J. C. Risset. J'ai aussi récupéré un fichier expliquant la façon dont les cordes et la caisse génèrent les sons et une explication succinte de la façon d'accorder une guitare [5]. Enfin, j'ai récupéré les valeurs des rapports fractionnaires qui existent entre les différentes notes de la gamme diatonique [6], rapports qui serviront à effectuer des transpositions de notes. Tous ces documents sont mis à dispositions des étudiants sur le serveur de l'IUT.

2) la couverture du programme: Non le programme ne sera pas couvert en totalité. Mais l'était-t'il avant? Lorsque $80 \%$ du programme est incompris par $66 \%$ des étudiants peut-on considérer qu'il est traité en totalité? En fait les TP permettent de traiter les fondamentaux suivants : calcul d'énergie d'un signal, FFT, autocorrélation, filtres transverses et récursifs, décimation, suréchantillonnage en concentrant les efforts sur les aspects fonctionnels, par exemple en ne traitant pas la synthèse des filtres (Matlab fait très bien la synthèse à partir de gabarits : utilisation de fdatool). Ça peut frustrer les théoriciens, mais pas les étudiants et libère du temps pour la vérification des synthèses obtenues (par l'analyse des fonctions de transfert visualisées par exemple) et pour réaliser des systèmes de traitement complets, ce qui est impossible dans un TP de $2 \mathrm{~h}$ si on cherche à maîtriser la compréhension totale de chaque bloc.

3) Les cinq TP et leurs articulations: On ne fait ici qu'une présentation succinte et très incomplète, le détail et l'ensemble des exploitations étant l'objet de la section suivante.

- TP1 : Comment caractériser un son de guitare? Ce TP permet en 2heures de présenter tous les outils de TNS dont on aura besoin dans les TP suivants pour constater les évolutions du signal après chaque transformation : analyse temporelle, fft, énergie (calculée dans l'espace temps et dans l'espace fréquence ...), autocorrélation.

- TP2 : Comment Synthétiser des sons de guitare? On reconstruira le son d'une corde en ajoutant au fondamental successivement des harmoniques et une enveloppe. On montre qu'il est très facile en changeant un paramètre de produire les autres notes. C'est un TP de "respiration" (après un TP1 assez lourd) qui permet sur un cas simple d'utiliser les outils du TP1.

- TP3 : Comment translater le spectre d'un signal? On illustre les filtres transverses en mettant en œuvre un filtre de Hilbert et on réalise un effet flange. 
- TP4 : Comment réaliser une chambre d'écho? On va illustrer les filtres récursifs en mettant en œuvre un filtre passe-tout. Le paramétrage de ce filtre permet de faire varier la durée de l'écho et le retard.

- TP5 : Comment compresser ou dilater un spectre? Rien de neuf dans ce dernier TP mais une bonne synthèse de l'ensemble de ce qui a été vu précédemment et donc une bonne révision ... avec la réalisation de l'effet de phasing à la clé.

On pourrait constater que les filtres utilisés sont très particuliers et peu représentatifs des filtres couramment utilisés. Je répondrai que le formalisme est le même (coefficients de la réponse impulsionnelle ou de la fonction de transfert, courbe de module et de phase) et que les passe-bas, passe-haut, etc sont par ailleurs largement utilisés dans le module télécom. La technique de translation de spectre par filtre de Hilbert utilisée dans le TP3 est à la base de nombreuses techniques de modulation et démodulation numériques.

Dans chaque TP, les algorithmes sont testés sur un signal sinusoïdal simple puis sur un ou plusieurs de guitare et finalement sur le signal MMG.

\section{OBJECTIFS DÉTAILLÉS DES CINQ TP OU L'ART DE DÉCALER LES SONS}

\section{A. TP1 : Caractérisation d'un son de guitare}

Ce premier TP permet de se familiariser avec différentes mesures de caractérisation de signaux. Les étudiants travaillent systématiquement à l'aide de deux signaux :

- un signal synthétique correspondant à l'échantillonnage à 44,1 KHz d'un signal sinusoïdal de fréquence $200 \mathrm{~Hz}$.

- une note de guitare réelle correspondant à un LA3 (440

$\mathrm{Hz}$ ) lui aussi échantillonné à 44,1 KHz.

La première partie du TP concerne l'analyse temporelle. Le signal est visualisé à différentes échelles de temps permettant d'observer l'enveloppe ou le contenu quasi-périodique. L'ordre de grandeur de la fréquence fondamentale et le nombre d'échantillons par période sont estimés visuellement. On observera fig.1 qu'un doute peut exister dans la détermination de la période ... Deux motifs ressemblants mais non identiques et séparés par des passages à zéro se succèdent. Rigoureusement ${ }^{2}$ la période correspond à la répétition du double motif, ce qui conduit à une fréquence de $220 \mathrm{~Hz}$ alors que l'oreille perçoit en début de morceau le $220 \mathrm{~Hz}$ puis le $440 \mathrm{~Hz}$ domine. Le réel est plus riche et plus intéressant que le synthétique... Il est prévu l'an prochain d'utiliser un logiciel simple de visualisation temps fréquence afin de mieux analyser les effets produits. les étudiants calculent ensuite l'énergie et la puissance moyenne $\mathrm{du}$ signal, fonctions qui seront bien utile pour ajuster le niveau de perception de signaux à comparer après leur transformation. Cette partie se termine par le tracé de la fonction d'autocorrélation qui permet de confirmer la valeur de la période du signal (localisation du premier pic) et la

\footnotetext{
${ }^{2}$ si on peut dire pour un signal quasi périodique
}

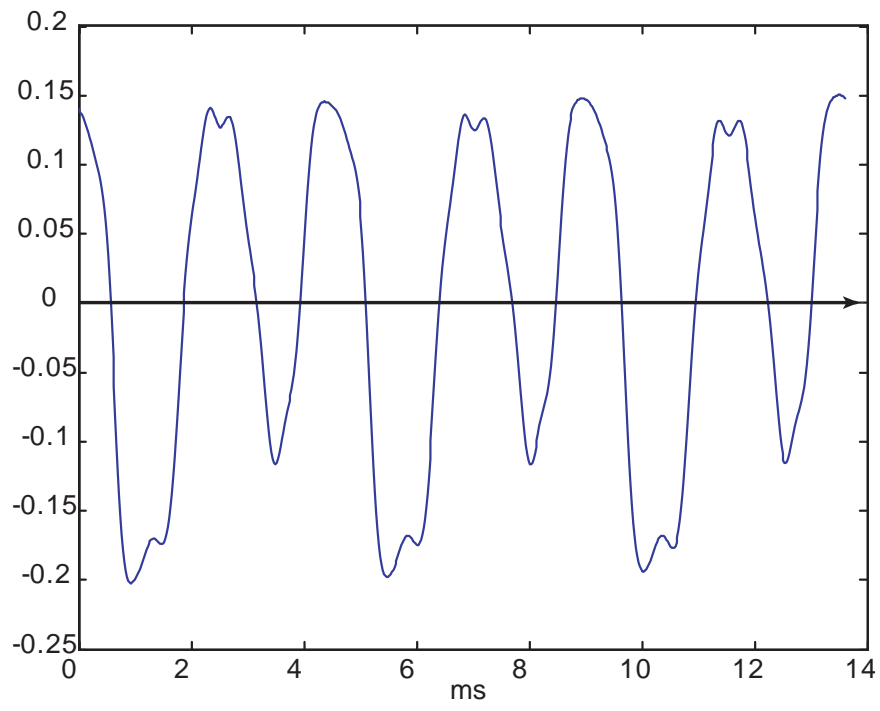

FIG. 1. Extrait des échantillons de la note LA3. La période correspond t'elle à $220 \mathrm{~Hz}$ ou à $440 \mathrm{~Hz}$ et quelle perception auditive en avons nous?

valeur de l'énergie $C_{x x}(0)$.

La seconde partie du TP est consacrée à l'analyse fréquentielle. Par le passé, un ensemble cours, TD, TP étaient consacrés à l'analyse spectrale : choix du nombre de points, de la fenêtre d'apodisation, etc. Les étudiants n'en retenaient rien! J'ai décidé de faire simple et de n'utiliser que la FFT avec fenêtre rectangulaire. Les étudiants déterminent le nombre de points pour obtenir une résolution de $0,5 \mathrm{~Hz}$ et constateront l'effet de l'ajout de zéros sur la résolution. On observe fig. 2 l'allure du spectre du LA3. Les étudiants sont invités à afficher en abscisse les fréquences vraies et à mesurer les amplitudes relatives et les fréquences des cinq raies les plus significatives. Ces mesures serviront dans le TP2 à synthétiser le son de guitare. Les étudiants visualisent aussi les spectres d'autres notes de guitare et repèrent les fréquences fondamentales.

Les étudiants les plus avancés sont invités à estimer la densité spectrale de puissance, la puissance moyenne et l'énergie à partir de la transformée de Fourier discrète.

Outre les aspects "mesures", le point le plus intéressant de ce TP est le débat sur la valeur de la fréquence fondamentale $220 \mathrm{~Hz}$ ou $440 \mathrm{~Hz}$ observé à la fois dans les analyses temporelle et fréquentielle. L'origine du phénomène est la mise en résonance de la caisse de la guitare (résonance à $220 \mathrm{~Hz}$ ).

\section{B. TP2 : Synthèses de sons de guitare}

Le premier TP est relativement difficile à cause du nombre relativement important de notions nouvelles et surtout parce que c'est le premier contact avec les signaux numériques. Le second TP est beaucoup plus simple et apparaît comme un TP ludique de respiration qui permet de resynchroniser les étudiants les plus lents avec les autres. A chaque étape du TP, les étudiants doivent écouter le signal synthétisé en comparaison du signal original LA3, l'observer dans les espaces 


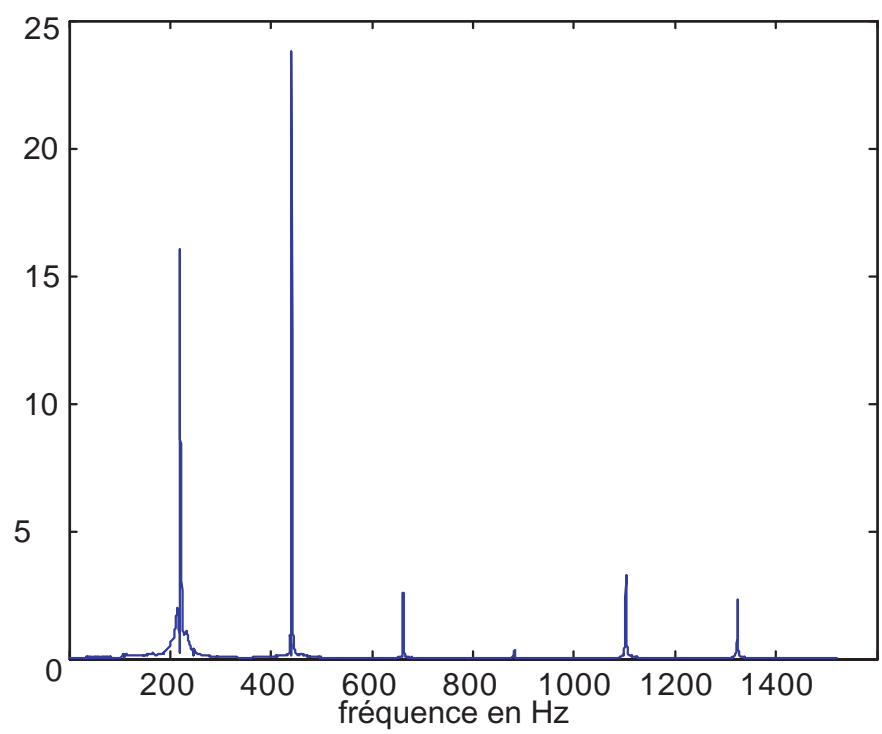

FIG. 2. Le spectre du LA3. On notera la prépondérance du $440 \mathrm{~Hz}$ sur le $220 \mathrm{~Hz}$

temporel et spectral après avoir ajusté l'énergie à celle du signal LA3 ... On note qu'on revisite l'ensemble des mesures vues au TP1. Le son synthétique est construit (et écouté) progressivement selon les étapes suivantes :

- construction d'un signal sinusoïdal d'amplitude 1 et de même fréquence que le LA3,

- ajout progressif des 4 harmoniques les plus significatives en tenant compte de leur poids relatif mesuré dans le TP1,

- modélisation de l'enveloppe du signal réel par une exponentielle décroissante dont on détermine la constante de temps,

- association de l'enveloppe synthétisée et du signal périodique de l'étape 2.

Enfin les étudiants sont invités à synthétiser de la même façon, à écouter et à comparer aux originaux le son des autres cordes. Il leur suffit de changer le paramètre fréquence fondamentale.

C. TP3 : Translations de fréquence : comment changer la hauteur d'une note, comment créer un battement fréquentiel?

Le battement est créé en ajoutant au son initial, une version du même son légèrement translaté en fréquence de $f_{t}$. On réalise ainsi un effet de modulation de l'amplitude à la fréquence $f_{t} / 2$. Un travail de réflexion est mené en TD avec les étudiants afin de montrer que même si l'objectif s'apparente à celui de la transposition de fréquence utilisée en radiocommunication à bande latérale unique, le procédé par multiplication et filtrage d'une bande latérale est inexpoitable ici parce que la valeur de la translation est inférieure à la bande passante du signal. On invite les étudiants à utiliser un procédé identique à celui de la modulation numérique MAQ utilisant deux porteuses en quadrature à ceci près qu'on ne dispose pas des voies d'entrées directe $i$ et quadrature $q$. On justifie ainsi la nécessité de produire une version déphasée de $\pi / 2$ du son original et par là même l'utilisation d'un filtre de Hilbert. Par l'étude et l'exploitation de ce filtre, on illustre la partie du cours relative aux filtres non récursifs. Le filtre de Hilbert a précédemment été étudié en TD. Dans la première partie du TP, on fournit aux étudiants les coefficients de la réponse impulsionnelle d'un filtre de Hilbert. Ils observent le module et la phase de la fonction de transfert de ce filtre afin de déterminer d'une part dans quelle zone de fréquence il est exploitable (ondulation limitée du module) et d'autre part discuter les conséquences de la linéarité de la phase. Un travail important d'explication avec démonstration sur un signal sinusoïdal simple est mené pour montrer que la pente de la phase traduit un retard (de $n_{0}$ échantillons) et que l'ordonnée à l'origine de la phase est responsable du déphasage de $\pi / 2$ introduit par le filtre à toutes les fréquences. Si on note $s$ le son original, st le son translaté spectralement et $f_{t}$ le décalage en fréquence, alors le son translaté est produit simplement par :

$s t(n)=s\left(n-n_{0}\right) \cdot \cos \left(2 \pi f_{t} n\right)-\operatorname{Hibert}[s(n)] \cdot \sin \left(2 \pi f_{t} n\right)$

On notera que la détermination du retard $n_{0}$ était indispensable pour compenser dans la voie cosinus le retard introduit par le calcul du filtre de Hilbert dans la voie sinus. Le signal original $s$ et le signal $s t$ sont finalement ajoutés pour produire l'effet musical recherché.

Une autre application est proposée en troisième partie de TP. En utilisant le même principe, en partant d'un LA3 $(440 \mathrm{~Hz})$, il est possible par une translation de $180 \mathrm{~Hz}$ de produire un RE\#4 $(620 \mathrm{~Hz})$. Les étudiants réalisent cette transformation et comparent le son produit à celui d'un son original RE\#4 (fichier fourni). Ils constatent la différence sonore et aussi les différences spectrales. En effet, pour transposer la note le spectre ne doit pas être translaté mais dilaté afin que les harmoniques subissent une translation de fréquence proportionnelle à leur rang.

Enfin, il est proposé en fin de TP, de synthétiser et d'utiliser un filtre réjecteur du $220 \mathrm{~Hz}$ et de ses environs qui permette de répondre à la question soulevée dans le TP1. Entendons nous un son à $440 \mathrm{~Hz}$ ou à $220 \mathrm{~Hz}$ dû à la résonance de la caisse de guitare?

D. TP4 : Retards temporels : comment créer une chambre d'écho, comment créer un battement temporel?

Le quatrième TP illustre les filtrages récursifs. On commence par refaire le filtre réjecteur du $220 \mathrm{~Hz}$ étudié en fin de TP3 et on constate que la forme récursive permet de réduire le nombre de coefficients du filtre à condition de renoncer à la linéarité de la phase. La seconde partie du TP consiste à construire deux versions d'une unité de réverbération, la plus simple correspond à des réflexions multiples entre deux murs (l'un étant situé près de la source $e$ ) avec effets d'atténuation (coefficient $g$ ) et de propagation (retard $r$ échantillons).

$$
s(n)=e(n)+g \cdot e(n-r) .
$$

La seconde unité étudiée est tirée de [7] et fait en sorte que le module de la fonction de transfert du filtre reste indépendant 
de la fréquence du signal d'entrée :

$$
s(n)=-g \cdot e(n)+e(n-r)+g \cdot s(n-r),
$$

ce qui correspond à un filtre passe-tout. Outre l'effet sonore manifeste, l'intérêt de cette étude est de travailler une nouvelle fois le lien entre les grandeurs réelles (temps de propagation du signal dans la salle hypothétique) et valeur numérique du retard en nombre d'échantillons, d'observer la réponse impulsionnelle qui fait nettement apparaître les échos et prend alors un sens physique évident, etc.

La fin du TP est consacrée à la réalisation d'un effet musical. On ajoute à un bruit blanc $b(n)$ une version décalée de $n_{0}$ échantillons :

$$
s(n)=b(n)+b\left(n-n_{0}\right) .
$$

Le décalage est choisi pour correspondre à la période d'un SOL3 $(784 \mathrm{~Hz})$. Les étudiants écoutent et observent le spectre et la fonction d'autocorrélation du bruit $b(n)$ et de $s(n)$ et apprécient la coloration du spectre. L'effet sur MMG est saisissant : le son semble tenu par une note de récitation. La coloration devenant d'autant plus évidente lorsque l'étudiant essaye aussi des décalages temporels correspondants à la période d'autres notes (RÉ\# ou SOL\#).

\section{E. TP5 : Dilatations de spectre : du LA3 au MI4, une seconde façon de réaliser un battement fréquentiel?}

Ce dernier TP va permettre de jongler avec les spectres. On utilise le plus souvent des associations de sous et sur échantillonnages pour conserver le spectre du signal alors que les fréquences d'échantillonnage à l'entrée et en sortie diffèrent. Si $F$ est la fréquence d'un signal échantillonné à $F_{e 1}$ et restitué à $F_{e 2}$ avec $F_{e 2} / F_{e 1}=p / q$, alors il faut suréchantillonner le signal numérique d'un facteur $p$ puis le souséchantillonner d'un facteur $q$. Le même traitement permet en maintenant la fréquence d'échantillonnage de passer la fréquence du signal de $F$ à $q / p \cdot F$.

La première application très simple consiste par suréchantillonnage $(\uparrow)$ d'un facteur 2 puis décimation $(\downarrow)$ d'un facteur 3 à transformer le son de guitare SOL $3^{\grave{e ̀ m e}}$ octave $(440 \mathrm{~Hz})$ en un MI $4^{\grave{e} m e}$ octave $(660 \mathrm{~Hz})$. Les étudiants constatent que contrairement à la tentative ratée de transposer le son par translation de fréquence (TP3), ici cela fonctionne parce que les harmoniques se trouvent translatées proportionnellement à leur rang. L'algorithme est extrêmement simple à produire (justification, tracés de spectre et calcul du filtre vus en TD) :

- insertion d'un échantillon nul entre chaque échantillon du signal initial,

- filtrage passe bas interpolateur de fréquence de transition $F_{c}=F_{e} / 6$

- extraction d'un échantillon sur trois.

La seconde application consiste à réaliser un effet appelé phasing. Il s'obtient en ajoutant au son initial, une version dont le spectre est très légèrement dilatée. Le coefficient de dilation choisi est de $442 / 440=1,0045$. Il produit un décalage de 2 $\mathrm{Hz}$ d'un son de fréquence initiale $440 \mathrm{~Hz}$. L'effet augmente l'ampleur du son initial ceci est très sensible appliqué au SOL de guitare ${ }^{3}$. La réalisation du son décalé s'obtient par la succession : $\uparrow 20$, filtrage $F_{c}=F_{e} / 26, \downarrow 13, \uparrow 11$, $F_{c}=F_{e} / 34, \downarrow 17$. La justification de cette décomposition est conduite avec les étudiants.

\section{DU VÉCU DE CES TP ET DES AMÉLIORATIONS}

\section{A. L'enquête : questions et résultats}

Un questionnaire de satisfaction a été soumis aux étudiants. Le quart d'entre eux seulement a répondu malgré l'automatisation (fichier pdf, cases à cocher, listes déroulantes,...). Il faut distinguer les réponses du groupe d'étudiants désirant s'orienter vers des études longues (e pour étude) de ceux envisageant une intégration professionnelle immédiate ou bien à l'issue d'une licence professionnelle (p pour professionnel).

- A la fin du module de TS de 30 heures, tous les étudiants (e) ayant répondu ont une image positive du TS et qui s'est améliorée pour les deux tiers d'entre eux. Alors que la moitié des (p) améliore leur appréciation du TS, un seul ayant au bout du compte une image plus négative qu'initialement.

- Tous les étudiants disent avoir compris l'importance du traitement du signal pour l'évolution des technologies.

- Tous les (e) pensent que le TS est utile à leur poursuite d'études et qu'ils l'utiliseront professionnellement. La moitié des (p) pense à juste titre l'utiliser dans leurs études et un seul d'entre eux professionnellement.

- Le tiers seulement des étudiants pense utiliser le TS pour leur usage personnel. Cela dénote encore une association réductrice du TS aux mathématiques en oubliant l'aspect fonctionnel. Par exemple, combien d'entre eux utiliseront un logiciel de retouche d'images et rééchantillonneront leurs photos?

- Une majorité des étudiants a jugé les TP difficiles mais en même temps motivants. Par contre, alors que les TD étaient construits pour préparer les TP, cela a été peu perçu par les étudiants bloqués trop souvent sur les premiers exercices d'application immédiate du cours.

- Etonnamment, les cours en amphi ont été jugé intéressants par la plupart (ce qui est en opposition avec une idée reçue sur les amphis) même si un petit tiers les a trouvé difficiles à comprendre.

- En moyenne les (e) ont consacré 15h de travail personnel au module de TS et $6 \mathrm{~h}$ à la préparation du devoir pour un résultat satisfaisant, alors que les (p), pourtant moins à l'aise n'ont consacré au module que $10 \mathrm{~h}$ de travail personnel et $3 \mathrm{~h}$ à la préparation du devoir pour un résultat très insuffisant.

\section{B. On peut toujours mieux faire}

Constatons d'abord que les principaux objectifs sont atteints. Un accroissement significatif de la motivation, et une

\footnotetext{
${ }^{3}$ Dans le même esprit, on associe trois cordes à une touche de piano qui seront infiniment peu désaccordées entre elles par l'accordeur. De la réussite de ce fin réglage résultera la qualité, l'ampleur, la chaleur du son produit par le piano.
} 
implication importante des étudiants préparant une poursuite d'études comme le montre leur investissement dans le travail personnel.

Il reste que deux points à mon sens conduiront à une évolution des enseignements. Tout d'abord l'apport du TS dans la vie courante doit être renforcé en multipliant les exemples d'application et les témoignages de professionnels au travers d'exposés d'études de cas traités sous l'angle fonctionnel. Le TS est encore trop associé aux mathématiques dans l'esprit des étudiants. Il faut tenter de leur montrer que l'outil informatique permet de répondre à leurs interrogations et qu'ils ont les compétences pour l'utiliser. Par exemple, les logiciels de retouche d'images mettent en oeuvre une variété de traitements qualitativement plus ou moins bien compris. On peut montrer en cours qu'il est relativement simple de produire des images tests révélatrices des conséquences de l'application de ces différents traitements...

Le second point est le remaniement des TD de façon à mettre en avant l'aspect fonctionnel et aussi en vue de produire un apprentissage autonome des méthodes de résolution par une décomposition extrêmement fine des questions des premiers exercices du TD. Expliciter et rédiger totalement le processus mental de résolution : quels sont les outils, les données de départ, les paramètres, l'objectif, le plan puis les détails de calcul (explicitation du passage d'une ligne à la suivante par le questionnement du TD, une seule transformation mathématique à la fois),etc. Bien entendu en cheminant dans le $\mathrm{TD}$, la finesse de description se réduira d'exercice en exercice. Et bien entendu, plus ce procédé sera mis en oeuvre tôt et intensivement dans la formation et plus la progression des étudiants devrait être significative.

\section{CONCLUSion}

J'espère vous avoir convaincu que l'on peut aider les étudiants qui galèrent à regagner la rive accrochés à un math de fortune! Et que le chant des sirènes, le bruit de la mer du mistral et des galets les motivent. Une fois sauvés et renforcés par le goût du succès et de l'effort produit, ils sont prêts pour un remathage sérieux leur permettant de repartir au long cours.

Le chercheur aussi a appris et savouré, que ce soit au sujet de la continuité de transmission historique entre le geste ancien de l'organum du IX ${ }^{\text {ème }}$ siècle et le phasing de nos synthétiseurs du XXI ${ }^{\grave{e} m e}$ siècle ou bien au sujet des ruptures liées à un simple changement d'échelle : un décalage de quelques centaines de Hertz produit deux sons clairement distingués alors qu'un décalage d'un dixième de hertz produit un son unique modulé ou enrichi. De quoi méditer ou provoquer un débat avec les étudiants ... Quelques uns des effets produits sont disponibles sur ce site [8].

\section{REMERCIEMENTS}

Je remercie Michel Lemeu pour le temps qu'il m'a consacré, pour son exceptionnelle aptitude à faire partager sa passion pour la musique et à initier. Ses idées, ses commentaires enrichissants, son aide ont été pour moi une source d'inspiration et de motivation tout au long de cette expérience.

\section{ANNEXE A : SUJET DU TP N ${ }^{\circ} 3$}

TRANSPOSITIONS DE FRÉQUENCE

\section{Objectifs du TP3}

- utiliser des filtres transverses dans le domaine temporel,

- observer les propriétés (module, phase) des filtres transverses numériques,

- mettre en oeuvre un filtre de Hilbert,

- transposer la hauteur d'un son réel.

\section{Caractérisation d'un filtre de Hilbert}

A- Chargez le fichier contenant la réponse impulsionnelle du filtre de Hilbert en utilisant la fonction load de matlab. On appelera hilb3 le vecteur matlab.

B- Observez les caractéristiques de ce filtre avec fvtool (hilb3) ou à défaut la fonction freqz.

1) Observez la réponse impulsionnelle

a) Quel est le nombre de coefficients de ce filtre ?

b) Quel est l'indice du coefficient du centre de la réponse impulsionnelle?

2) Réglez pour l'observation en fréquence la fréquence d'échantillonnage sur 44,1 Khz.

3) Observez le module de la fonction de transfert

a) Quelle est l'ondulation (en dB) dans la bande passante?

b) Quelle est l'amplification dans la bande passante et de combien fluctue t'elle en $\%$ ?

c) Quelles sont les limites basse et haute de la bande passante (on sort alors de l'amplitude d'ondulation calculée ci-dessus)?

4) Observez la phase de la fonction de transfert

a) Quelle est la valeur de la phase à $\omega=0$ ?

b) Mesurez très précisément la pente de décroissance de la phase (on pourra observer la vitesse de groupe $d \Phi / d \omega)$.

5) En supposant que le signal d'entrée est $e^{j k \omega}$, quel sera le signal de sortie si on suppose la caractéristique du module idéale?

C- Construire un signal stest : coswn contenant 44100 échantillons et résultant de l'échantillonnage d'un signal de $440 \mathrm{~Hz}$ à 44,1 Khz.

D- Filtrez ce signal par le filtre de Hilbert à l'aide la fonction filter de matlab (signal de sortie dtest). Comparez astucieusement la réponse temporelle de la sortie à celle de l'entrée sur environ 200 échantillons et en évitant les 100 premiers (transitoire de démarrage du filtre). Si vous avez le temps, refaites l'expérience avec stest issu de l'échantillonnage d'un signal à $1 \mathrm{Khz}$. 
E- Conclusion. Quel est le rôle de ce filtre?

Transposition d'un cosinus pur par filtrage de Hilbert

F- On veut translater tout le spectre d'un signal $x(n)$ de $10 \mathrm{~Hz}$ vers les hautes fréquences. On se préoccupe dans ce paragraphe d'un signal $x(n)$ simple : le signal stest construit à la question $C$. On s'appuie sur la formule :

$\cos (2 \pi f n) \cdot \cos \left(2 \pi f_{t} n\right)-\sin (2 \pi f n) \cdot \sin \left(2 \pi f_{t} n\right)$

$$
=\cos \left(2 \pi\left[f+f_{t}\right] n\right)
$$

où $f$ et $f_{t}$ sont les fréquences réduites (normalisées) $F_{\text {reelle }} / F_{\text {echant }}$ et où $f_{t}$ représente le décalage fréquentiel à réaliser. Il faut donc simplement synthétiser deux séquences $s t=\sin \left(2 \pi f_{t} n\right)$ et $c t=\cos \left(2 \pi f_{t} n\right)$ et obtenir $\sin (2 \pi f n)$ à partir de $\cos (2 \pi f n)$... ce qui est possible en faisant passer $\cos (2 \pi f n)$ dans le filtre de Hilbert.

Comme ce filtre (pour être réalisable) introduit un décalage temporel en sortie de $n_{0}$ échantillons, on modifie l'équation de réalisation comme suit :

$$
\begin{array}{r}
\cos \left(2 \pi f\left(n-n_{0}\right)\right) \cdot \cos \left(2 \pi f_{t} n\right) \\
-\sin \left(2 \pi f\left(n-n_{0}\right)\right) \cdot \sin \left(2 \pi f_{t} n\right) \\
=\cos \left(2 \pi\left[f+f_{t}\right] n-2 \pi f n_{0}\right)
\end{array}
$$

1) construire dtest par filtrage de hilbert de stest

2) construire un filtre qui réalise $y(n)=x\left(n-n_{0}\right)$; filtrer stest avec ce filtre (sortie srtest)

3) construire les signaux st et $c t$.

4) construire le signal transposé sttest.

5) Comparez les spectres de stest et de sttest. Vérifiez qu'il y a bien décalage de $10 \mathrm{~Hz}$. Observez aussi 200 échantillons de ces signaux.

\section{Effet spécial par battement fréquentiel}

On va dans ce paragraphe réaliser un effet spécial en combinant un son de guitare et ce même son décalé de $10 \mathrm{~Hz}$.

G- Chargez le fichier contenant un son de guitare en utilisant la fonction wavread de matlab. On appellera song le vecteur matlab.

H- De la même façon que dans le paragraphe précédent, réalisez :

$$
\begin{array}{r}
\operatorname{song}\left(n-n_{0}\right) \cdot \cos \left(2 \pi f_{t} n\right)-H i l b e r t[\operatorname{song}(n)] \cdot \sin \left(2 \pi f_{t} n\right) \\
=\operatorname{songt}(n)
\end{array}
$$

I- Comparez sur 44100 échantillons (après $0,5 \mathrm{~s}$ de signal) les spectres de song et songt. Ecoutez ces deux sons.

J- Ajoutez les sons song et songt et écoutez le résultat obtenu .

\section{Transposition de la hauteur d'un son de guitare}

$\mathrm{K}$ - On tente ici de créer le son d'un RE\# $(620 \mathrm{~Hz})$ à partir d'un LA $(440 \mathrm{~Hz})$. Exploitez les résultats du $\mathrm{G}$ et du $\mathrm{H}$ du paragraphe précédent pour obtenir resong correspondant au RE\# à partir de song.

L- Chargez le fichier contenant un son de guitare RE\#. Ecoutez ce son et comparez le à resong. Observez les spectres de ces deux signaux. Cette méthode est-elle la bonne pour construire le son de différentes cordes connaissant celui de l'une d'entre elle? Pourquoi?

\section{ANNEXE B : CORRIGÉ DU TP $\mathrm{N}^{\circ} 3$}

\section{TRANSPOSITIONS DE FRÉQUENCE}

Le code matlab apparaît en caractères télétype.

\%A load ('hilb3.mat');

\%B length (hilb3)

$1 \mathrm{a}$ : Le filtre possède 101 coefficients.

$1 \mathrm{~b}$ :L'indice du coefficient central est 50 (en partant de l'indice $0 \ldots)$.

fvtool (hilb3);

pause

3a : figure(3), ondulation de $\pm 0,165 d B$

$3 \mathrm{~b}:$ :Amplification unitaire à $1,9 \%$ d'ondulation.

$3 c$ : limites de bande passante $440 \mathrm{~Hz}$ et $21,6 \mathrm{KHz}$ (un peu juste côté bas pour l'application visée).

$4 \mathrm{a}:-90^{\circ} .4 \mathrm{~b}: n_{0}=-50$ par observation du tracé de la vitesse de groupe et $d \phi_{\circ} / d F=0.4082$ si on mesure la pente de la phase tracée en degré fonction des fréquences réelles. C'est le point le plus délicat du TP. La phase $\phi$ en radian vaut :

$$
\phi=-\frac{\pi}{2}-0.4082 \cdot F \cdot \frac{\pi}{180}
$$

avec $F$ fréquence réelle, $F_{e}$ la fréquence d'échantillonnage et $\omega$ la pulsation normalisée reliés par la relation :

$$
\omega=2 \cdot \pi \cdot \frac{F}{F_{e}}
$$

on obtient :

$$
\phi=-\frac{\pi}{2}-50 \cdot \omega
$$

$5: e^{j k \omega+\phi}=e^{j(k-50) \omega} \cdot e^{-j \frac{\pi}{2}}$. Soit un déphasage de $-90^{\circ}$ et un retard de 50 échantillons quelque soit $\omega$.

\%C stest $=\cos (2 * \mathrm{pi} . *(0: 44099) * 440 / 44100)$;

\%D dtest=filter (hilb3,1, stest) ;

\%F1 dtest=filter (hilb3,1, stest) ;

\%F2 r $=$ zeros $(51,1)$;

$r(51)=1 ;$ srtest $=f i l t e r(r, 1$, stest $)$;

\%F3 ct $=\cos (2 * \mathrm{pi} *(1:$ length (dtest) )

$\star 10 / 44100)$;

st $=\sin (2 * \mathrm{pi} *(1:$ length (dtest) $) * 10 / 44100) ;$

\% F4 sttest $=$ srtest . ${ }^{*}$ ct - dtest . ${ }^{*}$ st ;

\%55 figure (1);

plot (abs (fft (stest, 44100)), 'b'); hold on

plot (abs (fft (sttest, 44100)), ' $\left.r^{\prime}\right)$;

figure (2) ; plot (stest (200:400), 'b');

hold on, plot (sttest (200:400),'r');

\%G [song, $\mathrm{Fe}, \mathrm{bits}]=$ wavread ('A3.wav') ;

wavplay (song, 44100);

\%H dsong=filter (hilb3, 1, song) ;

srsong=filter $(r, 1$, song);

$\mathrm{ct}=\cos (2 * \mathrm{pi} *(1$ : length (dsong) $) * 10 / 44100)$;

$\mathrm{st}=\sin (2 * \mathrm{pi} *(1$ : length $(\mathrm{dsong})) * 10 / 44100)$;

songt=srsong . * Ct $^{\prime}-$ dsong . *st' ;

\%I figure (3)

plot (abs (fft (srsong (22050:66150)

,44100)), ' b') ;

hold on; plot (abs (fft (songt (22050:66150) 
Les spectres sont commentés sur la figure 4.

,44100)), 'r') ;

wavplay (songt, 44100 ) ;

\%J wavplay ( (song+songt ) /2,44100) ;

On perçoit un effet de battement de période $200 \mathrm{~ms}(10 \mathrm{~Hz} / 2)$ qui module l'amplitude du LA440. Il est facile de retrouver cela par le calcul dans le cas d'une entrée sinusoïdale ...

$\% \mathbf{K}$

Il faut tout simplement réaliser le décalage de fréquence qui ici vaut $620-440=180 \mathrm{~Hz}$. ct $620=\cos (2 * \mathrm{pi} *(1$ : length (dsong) )

*180/44100);

st $620=\sin (2 * \mathrm{pi} *(1$ : length (dsong) )

*180/44100);

songt =srsong . *ct620' - dsong . *st620';

\%L [resong, $\mathrm{Fe}, \mathrm{bits}$ ] =wavread ('D\#4.wav') ; wavplay (resong, 44100);

wavplay (songt, 44100);

figure (4);

plot (abs (fft (resong (22050:66150),44100))

,'b') ; hold on ;

plot (abs (fft (songt (22050:66150),44100))

' 'r') ;

On constate que le $440 \mathrm{~Hz}$ s'est bien décalé comme prévu à $620 \mathrm{~Hz}$. Ces deux raies de resong et songt sont bien superposées. Par contre les harmoniques translatées du 440 ne sont pas des harmoniques du 620, c'est pour cela que les ons produits sont si différents. Il aurait fallu non pas translater mais dilater le spectre, ce qui sera l'objet du TP5 qui mettra en œuvre sous et sur échantillonnage. Mais ceci est une autre histoire ...

\section{RÉFÉRENCES}

[1] M. Lemeu. (2004) Site personnel de michel lemeu. [Online]. Available : http ://pagesperso-orange.fr/michel.lemeu/

[2] P. Lalitte. (2002) Sud de jean claude risset : éléments d'analyse. [Online]. Available : http ://musique.ac-dijon.fr/bac2002/risset/

[3] J. C. Risset. (2002) Extraits mp3 de l'oeuvre sud de jean claude risset. [Online]. Available : ftp ://trf.education.gouv.fr/pub/educnet/musique/neo/04infos /formations/concours/baccalaureat/bac2002/sud/2sud.htm

[4] (2008) Acoustic guitar single notes. [Online]. Available : http ://www.freesamples-n-loops.com/free-guitar-samples.html

[5] (2004) Comment accorder une guitare ? d'après bac amérique du sud 2004. [Online]. Available : http ://www.chimix.com/an4/an40/bac/spe062.htm

[6] C. Sciences. (2003) Le son et la musique. [Online]. Available : http ://www.cap-sciences.net/upload/BM_musique.pdf

[7] E. Tisserand, J. Pautex, and P. Schweitzer, Analyse et traitement des signaux : méthodes et applications au son et à l'image. DUNOD, 2004.

[8] D. Demigny. (2008) Blog professionnel de didier demigny. [Online]. Available : http ://blogperso.univ-rennes1.fr/didier.demigny/

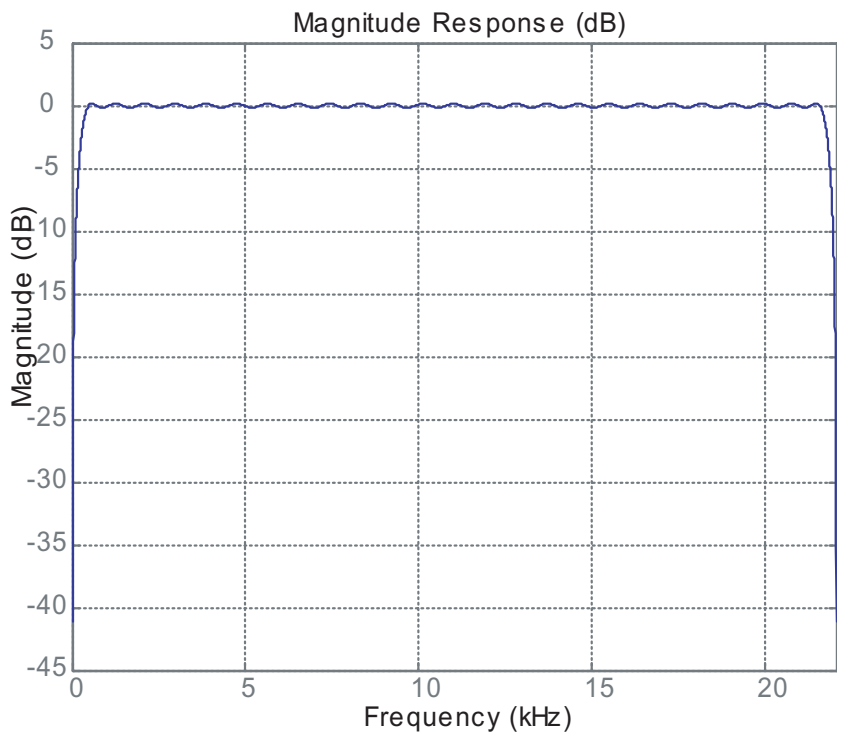

FIG. 3. Réponse en amplitude du filtre de Hilbert à 101 coefficients. On notera les limites haute et basse d'utilisation et l'ondulation dans la bande passante

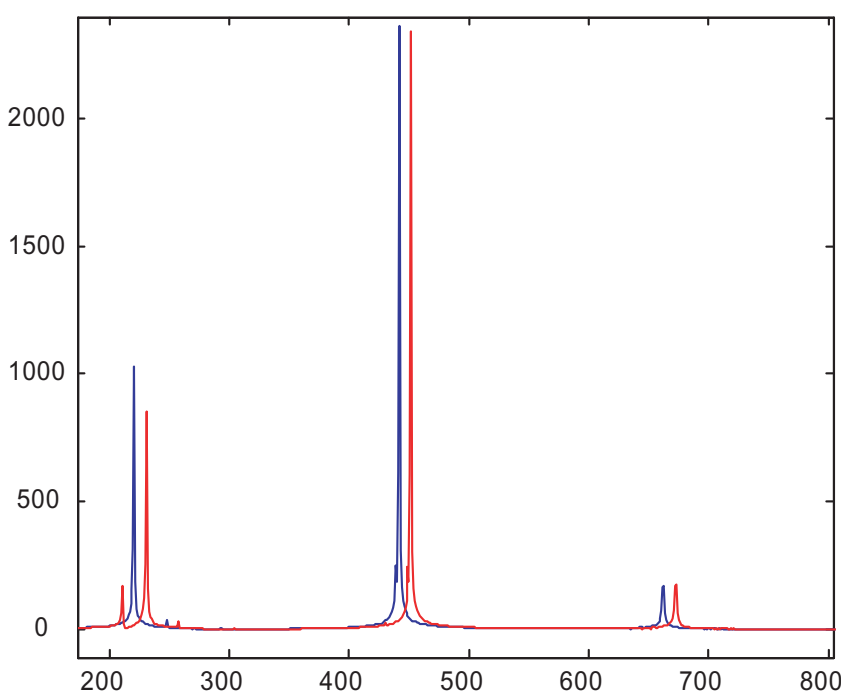

FIG. 4. Le spectre du LA3 original en bleu et le spectre translaté de $10 \mathrm{~Hz}$ songt en rouge. Les écarts d'amplitude des raies correspondantes des deux spectres très sensibles pour les basses fréquences proviennent de l'affaiblissement du gain en dehors de la bande passante du filtre de Hilbert. La limite basse à $440 \mathrm{~Hz}$ est un peu élevée et la raie à $230 \mathrm{~Hz}$ en rouge apparaît donc atténuée par rapport à la raie d'origine à $220 \mathrm{~Hz}$ en bleu. 